\title{
Therapeutic efficacy of phentolamine in the management of severe hand, foot and mouth disease combined with pulmonary edema
}

\author{
ZHIXIN YAN ${ }^{1}$, YAOKAI SHANG ${ }^{2},{\text { FENGXIAN } \text { LI }^{3}, \text { FEI XIE }^{1}, \text { HUILI QIAN }}^{4}$, YOUQI ZHANG $^{5}$ and BIN YUE ${ }^{1}$ \\ ${ }^{1}$ Department of First Pediatric Internal Medicine, Cangzhou Central Hospital; \\ Departments of ${ }^{2}$ Pediatrics and ${ }^{3}$ Internal Medicine, Yanshan Fude Hospital; Departments of ${ }^{4}$ Second and \\ ${ }^{5}$ Fourth Pediatric Internal Medicine, Cangzhou Central Hospital, Cangzhou, Hebei 061000, P.R. China
}

Received October 19, 2015; Accepted November 18, 2016

DOI: $10.3892 /$ etm.2017.4104

\begin{abstract}
The aim of this study was to examine the effects of phentolamine on severe hand, foot and mouth disease (HFMD) combined with pulmonary edema (PE). From May 2008 to December 2012, 53 children with severe HFMD plus PE were enrolled in the treatment group, receiving phentolamine intravenously at a loading dose of $5 \mu \mathrm{g} / \mathrm{kg} / \mathrm{min}$. The control group comprised 52 children with the same disease who did not receive phentolamine infusion. Data concerning creatine kinase (CK), CK-MB, cardiac troponin I (cTnI), heart rate, systolic blood pressure (SBP) and the duration of ventilation dependence and hospitalization were collected. Adverse events were also recorded. It was found that the phentolamine-treated patients exhibited significantly lower CK, CK-MB and cTnI levels, heart rate and SBP than the controls $(\mathrm{P}<0.01$ for all parameters). The average duration of ventilator dependence and hospitalization was significantly shorter $(\mathrm{P}<0.01)$ in the phentolamine group than in the control group. It was also found that the overall mortality rate was lower in the phentolamine group (5.8\%) than in the control group (11.5\%). No adverse events were observed in either group. Thus, these results offer preliminary evidence that phentolamine reduces mortality and relieves the symptoms of EV71-induced PE. Phentolamine is a potential therapeutic agent for this highly lethal disorder.
\end{abstract}

\section{Introduction}

Hand, foot, and mouth disease (HFMD) is an infectious disease, which is mainly induced by coxsackievirus A16 (CVA16) and enterovirus 71 (EV71), and commonly occurs in children $<5$ years old (1,2). Wang et al (3) indicated that cytokines are

Correspondence to: Dr Zhixin Yan, Department of First Pediatric Internal Medicine, Cangzhou Central Hospital, 16 Xinhua West Road, Cangzhou, Hebei 061000, P.R. China

E-mail: yanzhixin0610@sina.com

Key words: phentolamine, hand, foot and mouth disease, pulmonary edema the mediators of the severe systemic inflammatory response associated with EV71-induced pulmonary edema (PE). Several cases with HFMD are mild and reversible, while other cases exhibit rapid progression to neurogenic $\mathrm{PE}$ (4). PE is associated with a high early mortality rate of $30-40 \%$ (5). In the past three decades, Asian countries have experienced several widespread and severe outbreaks of HFMD with deaths predominantly occurring among children (6). At present, there are no satisfactory treatments for EV71-induced PE. Thus, more effective pharmacological strategies are urgently required to treat the neurological complications of this potentially fatal viral infection.

The underlying mechanisms of PE have been not completely elucidated due to its complex etiology. However, activation of the sympathetic nervous system, particularly the massive stimulation of $\alpha$-adrenoceptors following the discharge of catecholamines in response to endothelin receptor activation in the central nervous system, is considered to be the major factor resulting in cardiopulmonary dysfunction (7-9). Thus, blocking the activation of $\alpha$-adrenergic receptors is crucial for alleviating the symptoms of PE. Phentolamine is a reversible and nonselective $\alpha$-adrenergic antagonist (10). Krishnamoorthy and Weinberg (11) have indicated that phentolamine can attenuate or completely prevent epinephrine infusion-induced catecholamine release. Moreover, phentolamine has been indicated to competitively block $\alpha$-adrenergic receptors and decrease hypertension by antagonism of the catecholamines epinephrine and norepinephrine to further improve the cardiopulmonary function in patients with PE (12). These results are encouraging but not definitive since additional mechanisms may be involved. A former study has demonstrated that phentolamine only partly protects lung function, indicating that other mediators discharged during sympathetic activation might play important roles in increasing the permeability of the lung (13). Due to the lack of clarity of these results, the present study aimed to provide clarification.

Therefore, the present study tested the assumption that phentolamine alleviates the symptoms of HFMD combined with PE by attenuating the associated changes in cardiopulmonary function. In this study, preliminary evidence that phentolamine reduces mortality and relieves the symptoms of EV71-induced PE is provided. The favorable therapeutic outcome supports the potential of phentolamine as a candidate 
therapeutic agent for HFMD with PE. However, this requires evaluation in prospective clinical studies.

\section{Materials and methods}

Study design and ethics statements. This was a single center, open-label, randomized trial. The study was approved by and implemented under the guidelines of the ethics committees of Cangzhou Central Hospital (Cangzhou, China) as well as institutional review boards of Cangzhou Central Hospital. Randomization was conducted using a random digital table by assigning odd numbers to the phentolamine treatment group and even numbers to the control group. The investigators did not obtain prior notice of which participants were assigned to take either treatment. The next of kin of the children involved in this trial signed and provided written informed consent on the children's behalf prior to participating in the study.

Patients. Children in Cangzhou Central Hospital were recruited from May 2008 to December 2012. Eligible patients were required to have a clinical diagnosis with severe HFMD combined with PE based on the diagnosis and treatment guidelines for HFMD (2010) issued by the Ministry of Health of China (14). Children were excluded when they exhibited complications including chronic hepatitis, epilepsy, congenital heart disease, nephritis and serious metabolic or hematological diseases. Further exclusion criteria included chronic diarrhea, a history of allergy to the study drugs and participation in other clinical trials.

Intervention. All patients were treated with ventilation support using high levels of positive end expiratory pressure $(5-15 \mathrm{~cm}$ $\mathrm{H}_{2} \mathrm{O}$ ), as well as intravenous $\gamma$-globulin at a dose of $1 \mathrm{~g} / \mathrm{kg} /$ day for two days (Shandong Taibang Biological Products Co., Ltd., Taibang, China). In-patient children received methyl prednisolone at a dose of 3-5 mg/kg/time once daily for five consecutive days (Pfizer, Inc., New York, NY, USA). Furthermore, all patients were treated with mannitol to reduce intracranial pressure. Mannitol was administered at a dose of 0.5-1 g/ $/ \mathrm{kg} /$ time six times daily for seven consecutive days (Shandong Qidu Pharmaceutical Co., Ltd., Zibo, China). Concurrently, in the phentolamine treatment group, a phentolamine infusion (10 mg/ml; Shanghai Xudong Haipu Pharmaceutical Co., Ltd., Shanghai, China) was initiated at a dosage of $3 \mu \mathrm{g} / \mathrm{kg} / \mathrm{min}$ and titrated for the control of stress ulcer-related gastrointestinal bleeding during the physiological stress of HFMD. Additionally, phentolamine was administered intravenously to control the indices of blood pressure, heart rate and blood gas at a loading dose of $5 \mu \mathrm{g} / \mathrm{kg} / \mathrm{min}$. When the indicators of blood pressure, heart rate and blood gas stabilized, phentolamine administration was gradually decreased and then stopped within $24 \mathrm{~h}$. The dosage was adjusted from high to low as necessary to stabilize the vital signs. Therapy was continued for 3-5 days. Patients in the phentolamine treatment and control groups were followed up for 6 months.

Pathogen detection. Samples obtained from the stool, throat or cerebrospinal fluid were immediately sent to the laboratory of the Chinese Center for Disease Control and Prevention (Beijing, China) to confirm the association of EV71 infection with the illness by means of reverse transcription-polymerase chain reaction (RT-PCR).

Total viral RNA was isolated from the samples using TRIzol reagent (Invitrogen; Thermo Fisher Scientific, Inc., Waltham, MA, USA). Prior to RT-PCR, RNA samples were treated with DNase (Thermo Fisher Scientific Inc.). RT-PCR amplification was conducted using the following protocol: $10 \mu \mathrm{l} 10 \mathrm{X}$ PCR buffer (Thermo Fisher Scientific, Inc.), $3 \mu 1$ upstream primer, $3 \mu \mathrm{l}$ downstream primer and $8 \mu \mathrm{l}$ dNTPs (Thermo Fisher Scientific, Inc.), $1 \mu \mathrm{l} \mathrm{MgCl} 250 \mathrm{mM}$ (Thermo Fisher Scientific, Inc.), $1 \mu 1$ Taq DNA polymerase (Sigma-Aldrich; Merck Millipore, Darmstadt, Germany).

VP1-specific cDNA was synthesized and was used as a template for EV71 viral mRNA amplification using primers (EV2449-EV2780) with the sequences: Forward, 5'-GGAGATAGGGTRGCAGATGTAAT-3' and reverse, 5'-ATTTCC CAAGAGTAGTGATCGC-3'. $\beta$-actin was used as reference gene. For $\beta$-actin, forward: 5'-CTCCATCCTGGCCTCGCT GT-3' and reverse, 5'-GCTGTCACCTTCACCGTTCC-5'. The amplification reactions were as follows: Initial denaturation at $95^{\circ} \mathrm{C}$ for 5 mins, followed by 15 cycles comprising denaturation at $95^{\circ} \mathrm{C}$ for $35 \mathrm{sec}$, annealing at $50^{\circ} \mathrm{C}$ for $16 \mathrm{sec}$ and extension at $72^{\circ} \mathrm{C}$ for $35 \mathrm{sec}$, followed by 40 cycles consisting of denaturation at $95^{\circ} \mathrm{C}$ for $35 \mathrm{sec}$, annealing at $48^{\circ} \mathrm{C}$ for $16 \mathrm{sec}$ and extension at $72^{\circ} \mathrm{C}$ for $35 \mathrm{sec}$, and final extension at $72^{\circ} \mathrm{C}$ for $10 \mathrm{~min}$. Subsequently, RT-PCR-positive samples were confirmed by sequence analysis within the VP-1 region of EV71 using BigDye v3.0 Sequencing kits (Thermo Fisher Scientific, Inc.) as well as an ABI 3730 automated sequencer (Applied Biosystems; Thermo Fisher Scientific, Inc.).

Outcomes. Venous blood samples were collected from all patients who were treated by ventilation support for 6 and $72 \mathrm{~h}$. These blood samples were used to measure the levels of creatine kinase (CK), CK-MB and cardiac troponin I (cTnI). The investigator recorded results for CK, CK-MB and cTnI, in the two groups. In addition, heart rate and systolic blood pressure (SBP) in the two groups were recorded every $6 \mathrm{~h}$, until these indexes dropped to the normal level. Moreover, the ventilation time and the duration of hospitalization in these two groups were collected. In addition, the investigator used a standard adverse-event case report form to gather information about adverse events if they occurred. The investigator also assessed whether or not the side events were associated with the drug.

Statistical analysis. SPSS 13.0 software (SPSS, Inc., Chicago, IL, USA) was utilized for all statistical analyses. All results are expressed as the means \pm standard deviations. All categorical variables were compared using a paired Student's t-test. Continuous variables were compared by means of an unpaired Student's t-test. $\mathrm{P}<0.05$ was considered to indicate a statistically significant difference.

\section{Results}

Subject characteristics. Between May 2008 and December 2012, a total of 106 subjects were enrolled in this trial. However, one of these participants was excluded due to a congenital ventricular septal defect. Thus, a total of 
Table I. Outcomes for children with severe hand, foot and mouse disease plus pulmonary edema a randomized trial evaluating the efficacy of phentolamine.

\begin{tabular}{|c|c|c|c|c|c|c|}
\hline Groups & No. & $\begin{array}{c}\text { CK-MB } \\
(\mathrm{U} / 1)\end{array}$ & CK (U/l) & $\begin{array}{l}\text { cTnl } \\
(\mu \mathrm{g} / \mathrm{l})\end{array}$ & $\begin{array}{c}\mathrm{HR} \\
\text { (beats/min) }\end{array}$ & $\begin{array}{c}\text { SBP } \\
(\mathrm{mmHg})\end{array}$ \\
\hline Phentolamine treatment & 53 & $17.61 \pm 1.31$ & $120.53 \pm 6.32$ & $0.45 \pm 0.08$ & $140.00 \pm 30.21$ & $126 \pm 16$ \\
\hline Control & 52 & $28.12 \pm 3.98$ & $450.75 \pm 8.56$ & $0.95 \pm 0.05$ & $160.22 \pm 35.34$ & $140 \pm 20$ \\
\hline $\mathrm{t}$ & & -17.736 & -20.082 & -20.71 & -3.079 & -3.865 \\
\hline P-value & & 0.008 & 0.004 & 0 & 0.002 & 0.005 \\
\hline
\end{tabular}

CK, creatine kinase; cTnI, cardiac troponin I; HR, heart rate; SBP, systolic blood pressure.

Table II. Clinical course of children with severe hand, foot and mouse disease plus pulmonary edema treated with phentolamine or standard therapy.

\begin{tabular}{lccc}
\hline Groups & No. & Ventilator dependence (days) & Hospitalization (days) \\
\hline Phentolamine treatment & 53 & $3.41 \pm 1.72$ & $15.52 \pm 3.51$ \\
Control & 52 & $4.95 \pm 2.16$ & $18.51 \pm 8.56$ \\
t & & -3.596 & -5.462 \\
P-value & & 0.004 & 0.002 \\
\hline
\end{tabular}

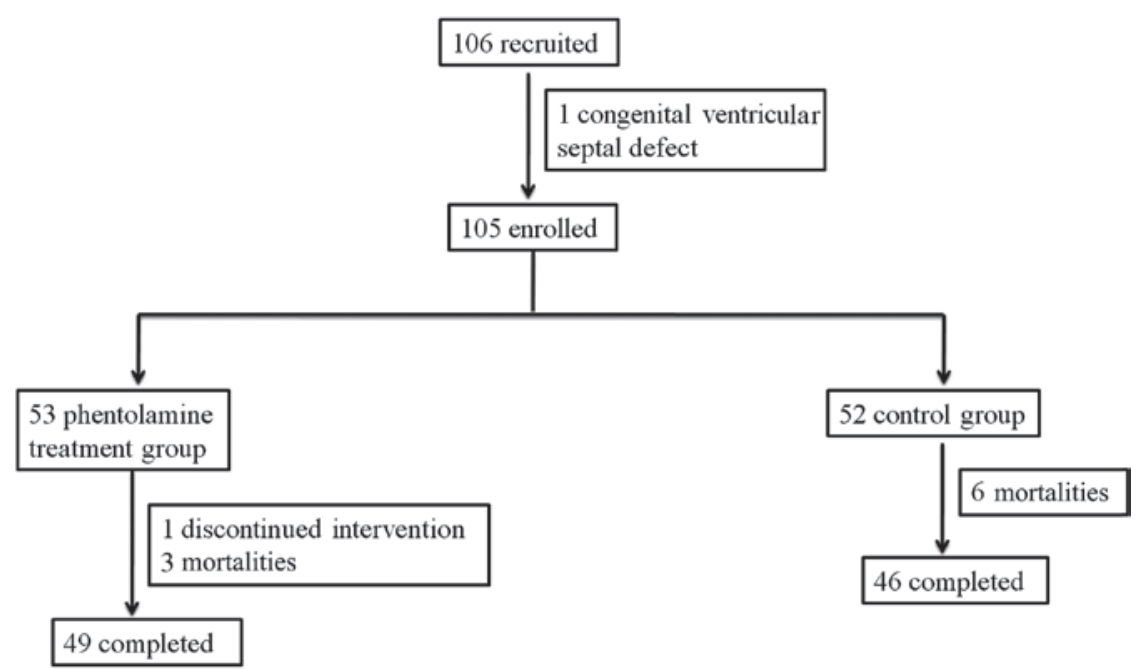

Figure 1. Flow chart of subjects with severe hand, foot and mouse disease combined with pulmonary edema in a randomized trial evaluating the efficacy of phentolamine.

105 patients were eligible and participated in the treatment, as shown in Fig. 1.

The baseline characteristics of the phentolamine and control groups were similar. Of the 105 patients, 53 were assigned to the phentolamine treatment group while 52 served as controls. Among the 53 patients of the phentolamine treatment group, $32(60.4 \%)$ were male and $21(39.6 \%)$ were female. The median age of the patients at disease onset was $1.6 \pm 0.8$ years, and 52 (98\%) patients were $<3$ years of age at the time of the study. Moreover, in the control group, $30(57.7 \%)$ were male and $22(42.3 \%)$ were female. The median age was $1.5 \pm 0.7$ years, and $50(96 \%)$ patients were $<3$ years of age. No significant differences were observed in gender, age and clinical disease degree between the two groups.

In addition, a total of $48(91 \%)$ and $51(98 \%)$ subjects tested positive for EV71 by RT-PCR and were confirmed to be acutely infected with EV71 in the phentolamine treatment and control groups, respectively.

Clinical outcomes. The phentolamine-treated patients exhibited significantly lower CK, CK-MB and cTnI levels, heart rate and SBP than did the control patients $(\mathrm{P}=0.004,0.008,0$, 0.002 and 0.005 for each parameter, respectively), as depicted in Table I. 
The key events during the period of hospitalization are summarized in Table II. The average durations of ventilator dependence and hospital time were shorter $(3.41 \pm 1.72$ vs. $4.95 \pm 2.16$ days; $15.52 \pm 3.51$ vs. $18.51 \pm 8.56$ days, respectively) in the phentolamine treatment group than in the control group. Significant differences were observed between the groups in requirement for mechanical ventilation and the duration of hospitalization $(\mathrm{P}=0.004$ and 0.002 for ventilation application time and the duration of hospitalization, respectively).

Safety. It was found that the overall mortality rate was lower in the phentolamine group ( 3 mortalities, $5.8 \%$ ) than in the control group (6 mortalities, $11.5 \%$ ). All other patients survived the 6-month follow-up. Other than these mortalities, no adverse events were observed in either group.

\section{Discussion}

The attributes of EV71-induced HFMD with central nervous system involvement are brain-stem encephalitis complicated by $\mathrm{PE}$ as well as a high mortality rate (15). There is no available antiviral treatment for EV71-induced disorders. We suggest that a more favorable outcome might result from blocking the stimulation of $\alpha$-adrenergic receptors to alleviate the symptoms of PE. Phentolamine, a reversible and nonselective $\alpha$-adrenergic antagonist, is recommended as an appropriate treatment to attenuate the vasoconstricting effects of excess catecholamine secretion (16). The present trial was designed to investigate the potential effects of phentolamine on severe HFMD combined with PE. The results indicate that phentolamine markedly decreased the mortality rate, and improved the cardiopulmonary functions in patients with this disorder.

Phentolamine has been utilized for the treatment of patients with PE (11). Phentolamine has been reported to induce relative hemodynamic stability $(17,18)$. Usually, the administration of $\alpha$-adrenergic blockers reduces and stabilizes blood pressure and heart rate by reducing circulating catecholamine levels and increasing intravascular volume $(19,20)$. Furthermore, $\alpha$-adrenergic blockers have inotropic and vasodilatory properties (21). In the present trial, it was demonstrated that phentolamine treatment significantly reduced the mortality rate, the average duration of ventilator dependence and duration of hospitalization, CK, CK-MB and cTnI levels, heart rate and SBP. Furthermore, a low incidence of side events in the phentolamine treatment group suggested that this drug may be a well-tolerated and safe therapy for children with HFMD plus PE.

The potential action mechanisms of phentolamine appear to be multifactorial. Firstly, EV71 may cause multiple organ damage (22). A previous study has indicated that phentolamine competitively blocks postsynaptic $(\alpha-1)$ and presynaptic $(\alpha-2)$ adrenergic receptors, thereby causing vasodilation and a reduction in peripheral resistance (23). Pharmacological effects of $\alpha$-blockers also include relaxation of vascular smooth muscle, and antihypertensive effects, via antagonism of the catecholamines epinephrine and norepinephrine, to further improve the cardiopulmonary function of patients with PE (12). Sympathetic blockade using phentolamine also prevents myocardial necrosis (24). Sympathetic hyperactivity is a primary characteristic of EV71-induced PE. Norepinephrine activation is thought to be the cause of sympathetic control of the heart, leading to increased contractility force and heart rate (25). The present observation of reductions in heart rate, CK levels and SBP following the infusion of phentolamine in patients with HFMD and PE supports this hypothesis.

Phentolamine, as a vasodilator, plays important roles in the reduction of blood pressure (26). It is worthy of note that the change in vascular tone associated with $\alpha$-blocker treatment necessitates fluid infusion to prevent the occurrence of considerable hypotension (27). In the current study, the infusion rate of phentolamine was maintained at a level of $5 \mu \mathrm{g} / \mathrm{kg} / \mathrm{min}$, so that its antihypertensive effect was relatively weak and its main role was in improvement of the microcirculation. Furthermore, no severe phentolamine-related hypotension or other side effects were evident in this study. Moreover, phentolamine treatment significantly lowered the overall mortality rate relative to that of the control group. This low incidence of side events of phentolamine further indicates that this drug is a well-tolerated and safe therapy for children with HFMD plus PE.

Several shortcomings in the current trial should be noted. The relatively small sample size limited the analysis. Additionally, eligible children were recruited in a single clinical center. Finally, the effects of phentolamine on cytokine levels were not performed. PE induced by EV71 is caused by aberrant cytokine activation that generates a systemic inflammatory reaction, which results in increased pulmonary vascular permeability (5). Due to these limitations, a randomized trial in multiple centers with a larger sample size, and more measurement indicators is required to identify the safety and effectiveness of phentolamine in the treatment of EV71-induced PE.

In conclusion, the present randomized study provides evidence that phentolamine markedly decreases mortality, and improves the cardiopulmonary functions in children with HFMD and PE induced by EV71. These favorable findings indicate that phentolamine has potential as a therapeutic drug for treating this disease. However, dose-response as well as time-course studies for phentolamine in animal models of EV71-induced HFMD with PE are further warranted.

\section{References}

1. World Health Organization (WHO): A guide to clinical management and public health response for hand, foot and mouth disease (HFMD). WHO, Geneva, 2011.

2. Cardosa MJ, Perera D, Brown BA, Cheon D, Chan HM, Chan KP, Cho $\mathrm{H}$ and McMinn P: Molecular epidemiology of human enterovirus 71 strains and recent outbreaks in the Asia-Pacific region: Comparative analysis of the VP1 and VP4 genes. Emerg Infect Dis 9: 461-468, 2003.

3. Wang SM, Lei HY, Huang MC, Wu JM, Chen CT, Wang JN, Wang JR and Liu CC: Therapeutic efficacy of milrinone in the management of enterovirus 71-induced pulmonary edema. Pediatr Pulmonol 39: 219-223, 2005.

4. Choi CS, Choi YJ, Choi UY, Han JW, Jeong DC, Kim HH, Kim JH and Kang JH: Clinical manifestations of CNS infections caused by enterovirus type 71. Korean J Pediatr 54: 11-16, 2011.

5. Wang SM, Lei HY, Huang MC, Wu JM, Chen CT, Wang JN, Wang JR and Liu CC: Therapeutic efficacy of milrinone in the management of enterovirus 71-induced pulmonary edema. Pediatr Pulmonol 39: 219-223, 2005.

6. Ma E, Lam T, Chan K, Wong $\mathrm{C}$ and Chuang S: Changing epidemiology of hand, foot, and mouth disease in Hong Kong, 2001-2009. Jpn J Infect Dis 63: 422-426, 2010. 
7. Fontes RB, Aguiar PH, Zanetti MV, Andrade F, Mandel M and Teixeira MJ: Acute neurogenic pulmonary edema: Case reports and literature review. J Neurosurg Anesthesiol 15: 144-150, 2003.

8. Theodore $\mathrm{J}$ and Robin ED: Pathogenesis of neurogenic pulmonary oedema. Lancet 2: 749-751, 1975.

9. Poulat P and Couture R: Increased pulmonary vascular permeability and oedema induced by intrathecally injected endothelins in rat. Eur J Pharmacol 344: 251-259, 1998.

10. Raja SN, Treede RD, Davis KD and Campbell JN: Systemic alpha-adrenergic blockade with phentolamine: A diagnostic test for sympathetically maintained pain. Anesthesiology 74 691-698, 1991.

11. Krishnamoorthy V and Weinberg G: Phentolamine for neurogenic pulmonary edema: Bench to bedside progress. Chest 142 : 809-810, 2012

12. Davison DL, Chawla LS, Selassie L, Tevar R, Junker C and Seneff MG: Neurogenic pulmonary edema: Successful treatment with IV phentolamine. Chest 141: 793-795, 2012.

13. Hirabayashi A, Nishiwaki K, Shimada Y and Ishikawa N: Role of neuropeptide $\mathrm{Y}$ and its receptor subtypes in neurogenic pulmonary edema. Eur J Pharmacol 296: 297-305, 1996.

14. Ministry of Health of the People's Republic of China: Guidelines for the diagnosis and treatment of hand, foot and mouth disease (version 2010). Beijing, 2010

15. Liu CC, Tseng HW, Wang SM, Wang JR and Su IJ: An outbreak of enterovirus 71 infection in Taiwan, 1998: Epidemiologic and clinical manifestations. J Clin Virol 17: 23-30, 2000.

16. Kobal SL, Paran E, Jamali A, Mizrahi S, Siegel RJ and Leor J: Pheochromocytoma: Cyclic attacks of hypertension alternating with hypotension. Nat Clin Pract Cardiovasc Med 5: 53-57, 2008

17. Pacak K, Eisenhofer G, Ahlman H, Bornstein SR, Gimenez-Roqueplo AP, Grossman AB, Kimura N, Mannelli M, McNicol AM and Tischler AS; International Symposium on Pheochromocytoma: Pheochromocytoma: Recommendations for clinical practice from the first international symposium. October 2005. Nat Clin Pract Endocrinol Metab 3: 92-102, 2007.
18. Kinney MA, Narr BJ and Warner MA: Perioperative management of pheochromocytoma. J Cardiothorac Vasc Anesth 16: 359-369, 2002

19. Duka I, Gavras I, Johns C, Handy DE and Gavras H: Role of the postsynaptic alpha(2)-adrenergic receptor subtypes in catecholamine-induced vasoconstriction. Gen Pharmacol 34: 101-106, 2000.

20. Sparks JW, Seefelder C, Shamberger RC and McGowan FX: The perioperative management of a patient with complex single ventricle physiology and pheochromocytoma. Anesth Analg 100: 972-975, 2005.

21. Osnes JB: Positive inotropic effect without cyclic AMP elevation after alpha-adrenergic stimulation of perfused hearts from hypothyroid rats. Acta Pharmacol Toxicol (Copenh) 39: 232-240, 1976.

22. Sun LM, Zheng HY, Zheng HZ, Guo X, He JF, Guan DW, Kang M, Liu Z, Ke CW, Li JS, et al: An enterovirus 71 epidemic in Guangdong Province of China, 2008: Epidemiological, clinical, and virogenic manifestations. Jpn J Infect Dis 64: 13-18, 2011.

23. van Zwieten PA: The influence of antihypertensive drug treatment on the prevention and regression of left ventricular hypertrophy. Cardiovasc Res 45: 82-91, 2000.

24. Neil-Dwyer G, Walter P, Cruickshank J, Doshi B and O'Gorman P: Effect of propranolol and phentolamine on myocardial necrosis after subarachnoid haemorrhage. Br Med J 2: 990-992, 1978.

25. Drummond G and Severson D: Cyclic nucleotides and cardiac function. Circ Res 44: 145-153, 1979.

26. Bek MJ, Wang X, Asico LD, Jones JE, Zheng S, Li X, Eisner GM, Grandy DK, Carey RM, Soares-da-Silva P and Jose PA: Angiotensin-II type 1 receptor-mediated hypertension in D4 dopamine receptor-deficient mice. Hypertension 47: 288-295, 2006.

27. McMillian WD, Trombley BJ, Charash WE and Christian RC: Phentolamine continuous infusion in a patient with pheochromocytoma. Am J Health Syst Pharm 68: 130-134, 2011. 